\title{
HispanismeS
}

Revue de la Société des Hispanistes Français

Pratiques artistiques dans l'espace public

\section{Les personnages du Quichotte dans l'œuvre et la correspondance de Sade}

Irene Aguilá-Solana

\section{(2) OpenEdition}

Journals

Édition électronique

URL : https://journals.openedition.org/hispanismes/471

DOI : 10.4000/hispanismes.471

ISSN : 2270-0765

Éditeur

Société des Hispanistes Français

Référence électronique

Irene Aguilá-Solana, «Les personnages du Quichotte dans l'œuvre et la correspondance de Sade », HispanismeS [En ligne], 14 | 2019, mis en ligne le 01 octobre 2019, consulté le 31 août 2021. URL: http://journals.openedition.org/hispanismes/471 ; DOI : https://doi.org/10.4000/hispanismes.471

Ce document a été généré automatiquement le 31 août 2021.

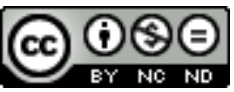

Les contenus de cette revue sont mis à disposition selon les termes de la Licence Creative Commons Attribution - Pas d'Utilisation Commerciale - Pas de Modification 4.0 International. 


\title{
Les personnages du Quichotte dans l'œuvre et la correspondance de Sade
}

\author{
Irene Aguilá-Solana
}

1 En tant que lecteur, Sade est un fin connaisseur des ouvrages de Cervantès; dans sa bibliothèque du château de La Coste, il possède les Nouvelles ${ }^{1}$ et le Quichotte ${ }^{2}$ et, dans ses lieux de détention, ces textes lui sont aussi chers. Le marquis, retenu au donjon de Vincennes, sa femme, Renée ${ }^{3}$, lui envoie deux volumes de l'auteur du Siècle d'Or, bien qu'elle n'en précise pas les titres, ainsi que les douze volumes du Nouveau Don Quichotte, comme elle l'écrit dans ses lettres du 26 mai et du 10 septembre $1781^{5}$. Trois mois plus tard, lorsque Renée se plaint du mauvais traitement que son mari inflige aux livres qu'elle lui a apportés, ce dernier titre est encore cité : « Surtout, quand les choses ne te conviennent pas, ne déchire ni m'écris dessus, parce que je paye tout ce que tu fais en ce genre et c'est de l'argent perdu. [...] le Nouveau Don Quichotte, il faut que je [le] paye $»^{6}$. Il dispose aussi des Nouvelles, en deux volumes, dans sa cellule de la Bastille en $1787^{7}$. Il s'agit très probablement de la traduction publiée par Coste d'Arnobat et Lefebvre de Villebrune en $1777^{8}$.

2 En tant qu'écrivain, Sade témoigne de son admiration pour le manchot de Lépante, comme le prouvent les passages explicites qu'il lui consacre dans Idée sur les romans (1799). Le roman du Quichotte - ouvrage immortel au succès universel--, tout comme les Nouvelles cervantines, qui couronnent définitivement l'écrivain $\mathrm{du} \mathrm{XVI}^{\mathrm{e}}$ siècle, lui semblent magnifiques :

Certes, si la chevalerie avait inspiré nos romanciers en France, à quel degré n'avaitelle pas également monté les têtes au-delà des monts! Le catalogue de la bibliothèque de Don Quichotte, plaisamment fait par Miguel Cervantès, le démontre évidemment ; mais quoi qu'il en puisse être, le célèbre auteur des mémoires du plus grand fou qui ait pu venir à l'esprit d'un romancier, n'avait assurément point de rivaux. Son immortel ouvrage, connu de toute la terre, traduit dans toutes les langues, et qui doit se considérer comme le premier de tous les romans, possède sans doute, plus qu'aucun d'entre eux, l'art de narrer, d'entremêler agréablement les aventures, et particulièrement d'instruire en amusant. Ce livre, disait Saint- 
Évremond, est le seul que je relis sans m'ennuyer, et le seul que je voudrais avoir fait. Les douze nouvelles du même auteur, remplies d'intérêt, de sel et de finesse, achèvent de placer au premier rang ce célèbre écrivain espagnol, sans lequel peut-être nous n'eussions eu, ni le charmant ouvrage de Scarron, ni la plupart de ceux de Lesage ${ }^{9}$.

Celui qu'il qualifie de célèbre auteur sans rival mérite tous ses compliments quant à l'influence exercée sur nombre d'écrivains français, parmi lesquels il se trouve ${ }^{10}$. Ce n'est pas donc notre but d'insister sur ce point. En revanche, notre analyse portera sur un aspect de l'écriture de Sade, tant dans ses textes fictionnels que dans ses écrits privés, où il manifeste cet attachement pour le chef-d'œuvre de Cervantès. Dans la présente étude, nous nous pencherons sur la présence des noms de quelques personnages du roman espagnol à travers l'antonomase, la métaphore ou la comparaison. Cet usage linguistique est fourni par le vocabulaire de l'époque ${ }^{11}$, attestant ainsi de l'importance de la réception de l'ouvrage cervantin en France. En effet, très tôt après la première traduction en langue française du Quichotte par César Oudin en $1614^{12}$, les répertoires ont recueilli « rossinante » (1633), " maritorne » (1642) et «dulcinée » (1648), comme le souligne Alexandre Cioranescu ${ }^{13}$. Puis, au XVIII siècle, le Dictionnaire comique (1718) de Le Roux explique que le mot "dulcinée» vient remplacer celui de «maitresse » et que, si on l'emploie avec un adjectif possessif ou un complément du nom, il prend une acception familière et moqueuse pour désigner une femme qui inspire des passions romanesques ${ }^{14}$. D'autres dictionnaires du temps n'intègrent plus tous ces noms, comme par exemple la $4{ }^{\mathrm{e}}$ édition du Dictionnaire de l'Académie (1762), ou leur donnent un sens différent ${ }^{15}$. Quant au Dictionnaire critique de la langue française de Féraud (1787-1788), nous y trouvons le mot « dom quichotisme ${ }^{16}$, tandis que le Dictionnaire de l'Académie française ajoute dans sa $5^{\mathrm{e}}$ édition (1798) "rossinante $»^{17}$ et "maritornes ${ }^{18}$. Enfin, des références à Cervantès et à sa création littéraire ont aussi leur place dans l'Encyclopédie ${ }^{19}$, un ouvrage que $\mathrm{M}^{\mathrm{me}}$ de Sade avait acheté sur la recommandation du marquis, lorsqu'il était prisonnier à Vincennes ${ }^{20}$.

Cependant un tel usage excède l'aspect purement linguistique chez l'auteur des Lumières pour devenir une ressource stylistique sur laquelle nous allons nous pencher dans cette étude. Parmi les noms issus de l'ouvrage cervantin qui étaient présents dans les dictionnaires de l'époque, seuls «Dulcinée », « don Quichotte » et "Sancho Pança » sont repris par Sade dans quelques-uns de ses romans et dans plusieurs missives qu'il échange avec ses proches. Arrêtons-nous sur chacun d'entre eux pour découvrir leurs particularités. "Dulcinée » conserve dans les textes du divin marquis quelques-unes des acceptions inscrites dans les répertoires lexicaux, à la seule différence que cette femme - qu'elle soit jeune, mûre ou âgée - y est souvent associée à des actions lubriques, style sadien oblige! Dans Aline et Valcour, l'antonomase de Dulcinée est riche en nuances. Le président de Blamont décrit Léonore de Kerneuil à son ami Dolbourg comme la « dulcinée ${ }^{21}$ de Sainville ${ }^{22}$ (soit M. de Karmeil). M. de Blamont qualifie la fille de «jolie aventurière " puisqu'elle réunit plusieurs facteurs qui la rendent une proie facile : elle est jeune, n'a pas de parents et a été portée disparue dans son pays. Elle est un exemple de «tout ce qu'il pleut par an de jeunes filles dans la province $»^{23} . \mathrm{M}^{\mathrm{me}} \mathrm{de}$ Blamont, pour sa part, raconte à Valcour les plans diaboliques de son mari envers cette demoiselle qu'elle croit vulnérable, qu'elle a hébergée et chérie en toute générosité. Néanmoins, dans son discours, le terme conserve une certaine nuance de mépris, lorsqu'on évoque l'illégitimité de la nouvelle venue :

Il [le président Blamont] a dit que je m'opposais depuis longtemps à un mariage très avantageux pour sa fille ; que par mes perfides conseils, j'empêchais cette fille de lui 
obéir, et que, joignant la ruse aux manœuvres ouvertes, j'ai été déterrer une petite créature avec laquelle l'ami qu'il destine à sa fille, a vécu, à la vérité quelques mois : que j'ai fait venir cette dulcinée dans ma terre, et qu'après l'avoir bien instruite, je la fais passer pour une fille à moi, enlevée par lui au berceau, dans l'abominable dessein de la prostituer à son ami; que par ce moyen, cet ami étant le même que celui dont il veut faire son gendre, ne peut plus maintenant le devenir, puisqu'il se trouverait alors avoir eu commerce avec les deux sœurs ${ }^{24}$.

Quand il donne des précisions sur l'argument du roman, Sade a encore recours à ce vocable qui décrit ici la femme avec laquelle un homme entretient des relations charnelles hors mariage :

[...] le président [de Blamont] faisait croire d'abord à Dolbourg que cette Sophie était la fille de sa maîtresse ; il faut se souvenir aussi que cette maîtresse était sœur d'une autre dulcinée, avec laquelle vivait Dolbourg, qu'ayant eu dans le même temps chacun une fille de ces maîtresses, ils s'étaient promis de se prostituer mutuellement ces deux enfants, quand elles auraient atteint l'âge nubile ${ }^{25}$.

Que soit présent de façon réitérée ce substantif « dulcinée » dans les lettres XXXVII, XLV et LII, qui encadrent l'épisode de Dolcini, le chirurgien qui soigna Léonore à Venise (épître XXXVIII), pourrait expliquer l'origine par allitération de ce patronyme. Néanmoins, alors qu'on aurait pu penser que le nom de Dolcini proviendrait de l'italien «dolce» (tout comme Dulcinée provient de l'adjectif espagnol «dulce»), il pourrait être aussi probable que ce nom dérive du toponyme Dolcigno ou Dulcigno, ville forte, dont les Vénitiens n'ont pas pu s'emparer en $1696^{26}$, et qui servait d'asile aux corsaires et aux brigands. Par conséquent, l'antonomase de la bien-aimée de don Quichotte définit, dans Aline et Valcour, une femme encline aux aventures galantes qui recherche les entreprises risquées, en se laissant guider par le hasard. Par ailleurs, nous pourrions envisager que le personnage cervantin, grâce à un petit jeu phonétique, ait servi au marquis pour nommer le chirurgien qui a aidé Léonore. La polysémie du mot « dulcinée » dans ce roman philosophique mettrait donc l'emphase sur un certain côté rebelle de Léonore et de Dolcini.

6 Tout au long des Cent Vingt Journées de Sodome, nous rencontrons quatre dulcinées assimilées à des prostituées ou à des esclaves, dont les prénoms ne sont indiqués que chez Rosalie et Lucile; les deux autres restent anonymes. Quant aux descriptions physiques de ces femmes, Sade en ébauche les traits indispensables pour créer un contraste (beauté / laideur, jeunesse / vieillesse), soit entre elles-mêmes, soit entre elles et leurs clients, ou pour additionner des caractéristiques diverses (vieillesse + saleté + laideur...). Leurs traits ne coïncident pas du tout : Rosalie, convoquée pendant la deuxième journée, est plutôt jeune - "pas plus de vingt-six ou trente ans » - et belle ${ }^{27}$; la doyenne de l'assemblée qui apparaît à la sixième journée est son antithèse : « une grosse et grande fille d'environ trente-six ans bourgeonnée, ivrognesse, jureuse et le ton poissard, et harangère, quoique d'ailleurs assez jolie ${ }^{28}$. Cependant toutes deux ont en commun l'obligation de satisfaire aux volontés de l'homme auquel elles sont livrées ou qui les paye. Dans le cas de la première dulcinée, elle doit se soumettre à la manie fétichiste de celui qui s'excite en manipulant sa chevelure :

Dès qu'elle entra, il la fit asseoir sur un tabouret très élevé et destiné à cette cérémonie. Aussitôt qu'elle y fut, il détacha toutes les épingles qui tenaient sa chevelure et fit flotter jusqu'à terre une forêt de cheveux blonds superbes dont la tête de cette belle fille était ornée. Il prit un peigne dans sa poche, les peigna, les démêla, les mania, les baisa, en entremêlant chaque action d'un éloge sur la beauté de cette chevelure qui l'occupait si uniquement. Il sortit enfin de sa culotte un petit vit sec et très roide qu'il enveloppa promptement des cheveux de sa dulcinée et, se 
manualisant dans le chignon, il déchargea en passant son autre main autour du col de Rosalie, et fixant sa bouche à ses baisers, il redéveloppa son engin mort ${ }^{29}$.

7 En ce qui concerne la doyenne, les fantaisies pour lesquelles elle est sollicitée sont la sitophilie (l'ingestion de nourriture en contact avec le corps) combinée avec la salirophilie (l'excitation sexuelle par la saleté) :

Cette même fille nous donna peu après le spectacle d'une fantaisie pour le moins aussi sale. Un gros moine, qui la payait fort bien, vint se placer à cheval sur son ventre ; les cuisses de ma compagne étaient dans le plus grand écartement possible, et fixées à de gros meubles pour qu'elles ne pussent varier. Dans cette attitude, on servit plusieurs mets sur le bas-ventre de la fille, à cru et sans qu'ils fussent dans aucun plat. Le bonhomme saisit des morceaux avec sa main, les enfonce dans le con ouvert de sa dulcinée, les y tourne et retourne et ne les mange qu'après qu'il les a complètement imprégnés des sels que le vagin lui procure ${ }^{30}$.

Les relations sexuelles de la huitième journée entre un grivois dans la soixantaine, dont le goût singulier le porte à " dépenser son argent avec des salopes de rebut " plutôt " qu'avec de jolies filles " ${ }^{31}$, et une femme, de plus de dix ans son aînée ${ }^{32}$, à l'aspect crasseux, mettent en scène une autre dulcinée. Sade se montre sarcastique lors de la description que la maquerelle Duclos fait de cette rencontre. Et pour cause. La maîtresse est "une vieille sorcière âgée de plus de soixante-dix ans ", une "emplâtre ${ }^{33}$, une "vieille garce ", une "duègne» au physique fort déplaisant: édentée à la "bouche infecte ", au "gosier putréfié » et au "cloaque infâme »; le « corps jaune et ridé, sec, pendant et décharné », ayant pour fesses "deux torchons ridés qui de ses hanches tombaient en ondulations sur ses cuisses ». La description burlesque de l'amant se résume à l'aspect risible de son sexe - «membre noir et ridé qui ne promettait pas de grossir de longtemps", "membre mort», " engin mollasse $»^{34}$ :

Il s'avance, toise sa dulcinée qui lui fait une profonde révérence : «Pas tant de façons, vieille garce, lui dit le paillard, et mets-toi nue [...] Alors notre homme s'approche et, saisissant sa tête, il lui colle sur les lèvres un des plus ardents baisers que j'aie vu donner de ma vie [...] et la bonne vieille, qui de longtemps ne s'était trouvée à pareille fête, les lui rendait avec une tendresse... qu'il me serait difficile de vous peindre ${ }^{35}$.

9 La dernière dulcinée des Cent Vingt Journées de Sodome est Lucile, une jeune fille aux ordres de la Duclos, qui, au cours de la quinzième journée, doit apaiser les désirs d'un vieux trésorier de France dont la «manie d'habitude, aussi sale que désagréable [...], consistait à chier sur le visage même de sa dulcinée, à lui barbouiller toute la face avec son étron et puis de la baiser, de la sucer en cet état ${ }^{36}$. C'est dans ce texte que le romancier fait le plus appel à l'antonomase pour désigner ce personnage cervantin. En outre, les traits qui caractérisent les dulcinées présentes dans le journal de bord de cette École du libertinage sont diamétralement opposés. Bien que toutes soient assimilées à des racoleuses ou à des esclaves sexuelles, elles s'opposent par leur physique : si la plupart sont repoussantes, d'autres sont jeunes et belles. Cette antinomie rappelle l'opposition patente entre la grossièreté d'Aldonza Lorenzo et les charmes attribués à Dulcinée du Toboso.

Dans Les Infortunes de la vertu, l'antonomase est aussi appliquée aux filles retenues par les moines lubriques du couvent de Sainte-Marie-des-Bois. Même si elles ont un prénom, que ce soit leur nom de baptême ou l'équivalent de leur nom de religion ${ }^{37}$, le substantif «dulcinée » sert aux gardiens de mot passe-partout pour les dénommer. Qu'elles s'appellent Omphale, Cornélie, Octavie, Florette ou Marianne, les prisonnières 
s'avèrent souvent interchangeables et facilement remplaçables. Raphaël, le moine qui garde le couvent, présente ainsi Marianne à Sophie ${ }^{38}$ : " cette dulcinée ${ }^{39}$ occupe dans une autre tour le même poste dont vous êtes honorée dans la vôtre $»^{40}$. De la même façon que Dulcinée du Toboso incarne pour don Quichotte la dame de ses pensées, alors qu'elle n'est qu'une simple paysanne, les récollets de Sainte-Marie-des-Bois jouissent aux yeux des habitants de la contrée d'un respect indu car ils sont considérés comme chastes et sobres alors qu'en réalité ils ne sont que luxure et intempérance. Sade se plaît habituellement à mettre en place des jeux de contrastes dans lesquels les individus ne sont pas tels qu'ils paraissent. Signalons enfin, pour ce qui est des écrits de fiction, que le terme « dulcinée » renforcé par l'adjectif « insolente » qui lui est associé, laisse transparaître chez Dolmancé, si friand des "délices de Sodome", dans le cinquième dialogue de la Philosophie dans le boudoir, sa haine contre celles qui, dans une certaine mesure, limitent le nombre de mâles prêts à s'adonner aux relations sodomites. Dans ce discours, grâce à la figure rhétorique dont il est question, le nom vient désigner une personne de sexe féminin, nettement inférieure à l'homme quels que soient son âge, son origine, ou sa condition : "Plats adorateurs des femmes, je les laisse, aux pieds de leur insolente dulcinée attendre le soupir qui doit les rendre heureux, et, bassement esclaves du sexe qu'ils devraient dominer ${ }^{41}$.

11 Dans la correspondance de Sade, nous retrouvons encore des exemples de la polysémie du terme. La marquise écrit à Gaufridy ${ }^{42}$, en mai 1777, à propos du caractère possessif de sa mère; elle nomme aussi l'une de ses sœurs, sans que l'on puisse dire avec certitude s'il s'agit de Françoise-Pélagie ${ }^{43}$ ou d'Anne-Prospère ${ }^{44}$ :

Elle [ $\mathrm{M}^{\text {me }}$ de Montreuil] a une patience d'ange et, pourvu qu'elle contemple sa belle Dulcinée $^{45}$, elle est satisfaite. Une fois sortie de ses pattes, j'aimerais mieux labourer la terre que d'y retomber. Elle ôte tout le mérite des services qu'elle rend par les vexations dont elle les accompagne ${ }^{46}$.

La marquise emploie le nom de la dame idéalisée par don Quichotte sous la forme d'une métaphore qui ferait allusion à ce que Françoise-Pélagie ou Anne-Prospère sont devenues dans l'imagination de leur mère après le travail de sape mené par celle-ci. Cette image de femme parfaite se profile dans d'autres lettres mais sous une perspective masculine car, après son évasion du 16 juillet 1778, Sade écrit à Gaufridy depuis son refuge de La Coste pour lui raconter un fait qui l'amuse: "Il y a une aventure unique entre Reinaud ${ }^{47}$ et moi : une lettre de moi, envoyée par lui à ma dulcinée au miroir, la lettre interceptée dans la prison et Reinaud publiquement nommé le messager des dieux. " (s.d. [un dimanche d'été], 1778). Cette « dulcinée » est $\mathrm{M}^{\mathrm{me}}$ Doyen de Baudoin. Il paraît que le reflet dans un miroir à la Conciergerie d'Aix aurait allumé l'étincelle d'une idylle platonique entre le marquis et cette jeune prisonnière ${ }^{48}$. Une semaine après s'être évadé, il reçoit une lettre de sa part où $\mathrm{M}^{\mathrm{me}}$ Baudoin se révèle absolument dévouée à celui qu'elle nomme "son ange gardien » ${ }^{49}$. Malgré le ton plaisant dont le marquis se sert pour narrer cette situation, il prie Reinaud de remettre à la " pauvre prisonnière » la quantité de sept livres dix sols. Cette disposition fut accomplie puisque l'article \# 45, qui fait partie du compte général de l'administration que Gaufridy eut des affaires du marquis pour l'année 1778, précise que l'on a "payé d'ordre de M. de Sade à une prisonnière à Aix, sept livres dix sols, suivant la lettre du 8 août $1778 »^{50}$.

Toujours au sein de sa correspondance, même si Sade n'y associe pas le nom de MarieDorothée de Rousset à Dulcinée, il existe des critiques littéraires qui reprennent 
l'antonomase de ce personnage cervantin pour se référer à cette amie d'enfance du marquis. Nous pouvons le voir, par exemple, à l'occasion d'une épître de Gaufridy à son confrère Ripert du 13 novembre 1783, dans laquelle il l'entretient de plusieurs sujets, entre autres de la santé de cette femme, atteinte de tuberculose, qui aurait été, d'après une note de Laborde, une de ses anciennes maîtresses: "à moins que le notaire, l'amoureux éconduit de Mademoiselle de Rousset, ait cessé de s'intéresser aux besoins et aux maux de son ancienne Dulcinée $»^{51}$.

Complétons les réflexions qui précèdent au sujet du personnage de Dulcinée en rappelant que celle-ci, vue par don Quichotte comme une jeune fille spirituelle, était en fait Aldonza Lorenzo, une paysanne décrite par Sancho comme une fille rustique, voire même une prostituée. C'est dans Aline et Valcour que Leónore rencontre, au fil de ses aventures, une bohémienne appelée Aldonza. Elle appartenait à une bande de brigands et avait voulu débaucher Clémentine en l'engageant à aller chez un homme à Tolède : «Bon, bon, répondit Aldonza, il ne faut pas être si difficile dans notre métier [...]. Cette Aldonza était à la vérité la plus corrompue de la troupe, il s'en fallait bien que nous [Léonore et Clémentine] eussions jamais rien entendu de pareil avec ses compagnes " ${ }^{52}$. Il est clair que le prénom de la vilaine «de muy buen parecer» du texte cervantin désigne bel et bien, dans le texte sadien, une prostituée qui avait « de vingt à trente ans » et était dotée "de [la] fraîcheur, et de [la] santé »53. À titre de curiosité, on rappellera qu'en France Aldonze ${ }^{54}$ était un prénom habituel en Provence parmi les nouveau-nés des deux sexes ${ }^{55}$ et que Sade, membre de la noblesse provençale, aurait dû le recevoir à son baptême. Cependant, malgré le souhait du père qui voulait le baptiser du nom de François-Aldonse-Donatien-Louis, «le vicaire de Saint-Sulpice, peu accoutumé aux lyriques consonances de la Provence, berceau de la famille, l'inscrit au registre des baptêmes sous un prénom erroné: Alphonse $»^{56}$. De toute évidence, l'écrivain tient beaucoup à ces prénoms, car Gaufridy rédige, en 1774, une pièce qui contient le nom complet de "messire Louis-Donatien-Adolphe Aldonce, marquis de Sade, chevalier, seigneur de la Coste, Mazan, Saumane et autres places ${ }^{57}$. De la même façon, dans une lettre d'ester à droit ${ }^{58}$ du 27 mai 1778, qui permet au marquis de se pourvoir contre l'arrêt du parlement de Provence, on s'adresse à lui par les prénoms qu'on aurait souhaité lui imposer à sa naissance: «Notre cher et bien aimé LouisAldonce-Donatien, marquis de Sade $»^{59}$. Plus tard, le marquis, se prévalant de ses mêmes noms de baptême, les mentionne dans un acte de protestation ${ }^{60}$ adressé au commissaire Chenon ${ }^{61}$ le 9 juillet 1789 , pour se plaindre d'avoir été transféré de la Bastille à Charenton, un asile pour aliénés parmi lesquels il se sent très mal à l'aise : "Moi, Louis Aldonze Donatien ${ }^{62}$, comte de Sade, mestre de camp de cavalerie et lieutenant général des provinces de Bresse, Bugei, Gex et Valromei » ${ }^{63}$. Puisque la bohémienne Aldonza était " une créature vraiment libertine ${ }^{64}$ le choix de ce prénom ne serait-il pas un clin-d'œil à la renommée même du marquis ?

Comptant beaucoup moins d'occurrences que l'emploi du nom de Dulcinée, citons celui de don Quichotte dont nous n'avons trouvé qu'une allusion dans la production romanesque de Sade. Elle se trouve dans le premier dialogue de La Philosophie dans le boudoir où le chevalier de Mirvel avoue à sa sœur que, bien que les femmes lui plaisent, il ne refuse pas les avances sexuelles de quelques hommes. Il justifie son comportement à partir des thèses matérialistes contemporaines - «l'homme est-il le maître de ses goûts? Il faut plaindre ceux qui en ont de singuliers, mais ne les insulter jamais, leur tort est celui de la nature $»^{65}$ - et critique les individus étroits d'esprit. Ceux-ci s'érigent en défenseurs dérisoires de ce que le sodomite appelle « droits ordinaires », c'est-à-dire 
qui s'inscrivent dans la vulgarité, par opposition au raffinement censé caractériser les pratiques libertines :

Un homme vous dit-il d'ailleurs une chose désagréable en vous témoignant le désir qu'il a de jouir de vous? non sans doute, c'est un compliment qu'il vous fait; pourquoi donc y répondre par des injures ou des insultes? Il n'y a que les sots qui puissent penser ainsi, jamais un homme raisonnable ne parlera de cette matière différemment que je ne fais; mais c'est que le monde est peuplé de plats imbéciles qui croyent que c'est leur manquer que de leur avouer qu'on les trouve propres à des plaisirs, et qui gâtés par les femmes, toujours jalouses de ce qui a l'air d'attenter à leurs droits, s'imaginent être les Doms Quichottes de ces droits ordinaires, en brutalisant ceux qui n'en reconnaissent pas toute l'étendue ${ }^{66}$.

Comme nous l'avons vu précédemment dans ce "dialogue philosophique», Sade attribue des défauts, tels que la sottise ou la déraison, au chevalier servant et à celle qu'il avait prise pour une noble dame, défauts du même ordre que ceux employés dans le roman, pour dénigrer les hommes hétérosexuels (" plats», « imbéciles»). On dirait que, dans le discours libertin, les « Doms Quichottes » et les « dulcinées» sont couverts de ridicule à cause de leurs goûts triviaux et de leurs habitudes traditionnelles.

Si la présence de l'antonomase de don Quichotte est minimale dans ses romans et au fil de sa correspondance, Sade a recours au nom du chevalier, parfois associé à celui de l'écuyer Sancho Panza, pour se référer à lui-même ou à d'autres individus. Ainsi, en 1776 (s.d.), répond-il aux accusations que Treillet, un tisserand de Montpellier, mentionne dans un mémoire à son encontre. Cet homme était le père de Catherine, une jeune fille qui travaillait comme cuisinière au château de La Coste où on l'appelait Justine. Il avait appris que l'employeur de sa fille était le marquis débauché et crut qu'elle était retenue contre sa volonté dans la maison de l'aristocrate. Selon Sade, cet individu avait agi d'une manière irrationnelle car, sous l'influence d'autres personnes qui l'auraient manipulé, il se serait imaginé des choses fausses. Il met donc Treillet sur le même plan que don Quichotte parce que, comme celui-ci, il est un insensé qui ne s'aperçoit pas de la réalité qui est sous ses yeux.

[c] et homme est visiblement un fourbe et un homme de mauvaise foi [...], ce coquinlà, qui est soufflé, se retournerait sur les prétendues insultes faites à ces gens qui sont venus, dont vous voyez bien qu'il est le don Quichotte, car qu'a de commun cette calomnie avec l'affaire de sa fille ?67

Quelques années plus tard, le marquis se compare au célèbre hidalgo ${ }^{68}$, fameux redresseur de torts, lorsqu'il prend la défense du style poétique de Milli ${ }^{69}$ de Rousset. Il répond le 12 mai 1779 à la lettre de son amie de l'enfance; celle-ci se plaît à composer des vers qu'elle soumet au jugement de l'illustre prisonnier: "Oui ma petite bête, comme un nouveau Don Quichotte, j'irais briser des lances pour apprendre aux quatre coins de l'univers que ma petite bête est de toutes les petites bêtes femelles respirantes entre les deux pôles, celle qui écrit le mieux et qui [est] la plus aimable $»^{70}$.

La mention de ce personnage cervantin interprété comme un grand fou se répète dans une missive de Carteron ${ }^{71}$; dans ce cas, ce n'est pas le marquis qui fait écho au chevalier espagnol mais le valet. En effet, celui-ci se défend des railleries que son maître aurait faites à propos de son accoutrement: «[...] quant à ma coiffure, ce n'était point un gauffrier comme vous le dites, mais seulement le moule à fromage ${ }^{72}$ que mon cousin Donquichotte avait sur sa tête lors de son combat contre le chevalier de la triste figure ${ }^{73}$ à la Montagne noire $»^{74}$. Il se peut que La Jeunesse ressemble physiquement au chevalier errant - du moins, c'est ce que pense le marquis -, et que cette similitude le pousse à créer une parenté métaphorique entre eux. La comparaison établie entre le subalterne 
de Sade et le célèbre hidalgo est à nouveau évoquée dans la réponse que le marquis reçoit la semaine suivante. De plus, comme son maître le compare à don Quichotte, La Jeunesse se permet l'audace d'établir un parallèle entre l'aristocrate et Sancho Panza du fait de la mauvaise calligraphie de son maître. Le duo Quichotte-Sancho est donc réuni dans la lettre que le valet adresse au marquis le 29 septembre 1779 (reçue le 4 octobre) :

Comme diable vous vous moquez de moi, Monsieur, mais vous avez bien raison, car j'ai bien peu d'érudition; j'aurais grand besoin que vous me donnassiez quelques leçons, enfin je suis eu, en attendant.

Permettez-moi aussi, Monsieur, de vous faire aussi une petite comparaison. Vous allez dire tout de suite que c'est bon pour moi, mais qu'importe, vous voudrez bien m'excuser d'autant mieux que c'est digne de moi. J'ai cru que vous auriez fait ce que vous vous seriez appliqué un peu dans l'art d'écrire. Mais je vois que c'est toujours tout de même, il semble que ce soit un essaim d'abeilles qui ait pâturé sur votre papier, à tout le moins. Si vous me comparez à mon cousin Domquichotte pour la figure, vous me permettrez, s'il vous plaît, de vous comparer à Sancho pour l'écriture. Je sais que jadis vous m'avez dit qu'il n'y avait que le menu peuple qui devait savoir écrire. Pas de colère surtout, pas de colère, vous pourriez me dire que je me donne des airs qui ne me conviennent pas, mais je vous en ai demandé pardon d'avance $^{75}$.

Soulignons enfin que les plaisanteries de Carteron qui associent le marquis à Sancho Panza ne sont pas les seules puisque, le 22 mars de la même année [1779], Sade s'identifie aussi à l'écuyer de don Quichotte. Dans ce cas, la dérision cède la place au sarcasme car Donatien, à la Bastille, se voit tel Sancho, devenu gouverneur de l'insula Barataria, et s'imagine donc trompé par tous ceux qui l'entourent. Il se méfie même de $\mathrm{M}^{\text {lle }}$ de Rousset et, en vérité, il n'a pas tort, car Milli essaiera de tirer profit de l'incarcération de son ami.

[...] les bouffonneries ou les affaires : ce qui est synonyme à présent pour moi, car vous sentez bien que quand on me parle d'affaires ici [à Vincennes], ce n'est qu'une pure bouffonnerie, c'est Sancho Pansa dans son île, à qui on fait croire que tout le monde attend ses ordres. C'est un petit persiflage auquel, soit dit sans la moindre rancune, vous vous prêtez comme au reste ${ }^{76}$.

En somme, l'analyse développée dans ce travail permet de mettre en relief deux questions principales. La première est l'intérêt porté par Sade au chef-d'œuvre de Cervantès car il se sert des noms de trois personnages cervantins (Dulcinée, don Quichotte et Sancho Panza) dans ses romans et dans sa correspondance, le plus souvent, au moyen de l'antonomase et, de manière secondaire, en guise de métaphore ou de comparaison. Il est utile de rappeler que quelques répertoires du Grand Siècle recueillent ces mots nouveaux; puis, au Siècle des Lumières, les dictionnaires de Le Roux, de Féraud, de Trévoux ou de l'Académie française conservent parfois certains de ces substantifs ou en rajoutent d'autres tirés du même ouvrage. Ce fut sans doute grâce à l'introduction de ces néologismes dans la langue française du XVII ${ }^{e}$ et du XVIII ${ }^{e}$ siècle, que Sade, par ses lectures du Quichotte et des Nouvelles de Cervantès, en vint à utiliser ces différents tropes. Par conséquent, le deuxième point abordé dans notre étude est la reconduction des qualités sémantiques dont ces noms sont dotés dans ses écrits. À partir des éditions des romans de Sade établies sur des manuscrits originaux, nous avons constaté une présence - assez discrète, il est vrai - d'antonomases tirées du Quichotte, chef-d'œuvre du Siècle d'Or. Il est possible de trouver de façon récurrente le signifiant « dulcinée » dans Les Cent Vingt Journées de Sodome (1785), Les Infortunes de la vertu (1787), Aline et Valcour (1795) et La Philosophie dans le boudoir (1795). Bien que 
l'acception de "dulcinée » proposée par les répertoires offre une définition claire et déterminée, le mot est polysémique chez Sade. En effet, les personnages féminins ainsi nommés reçoivent dans les mêmes textes d'autres désignations telles que "fille", "demoiselle ", " héroïne", " pécore ", " catin ", "garce» ou "putain». Quant à l'antonomase de don Quichotte, nous ne l'avons repérée que dans La Philosophie dans le boudoir où elle a le sens de chevalier médiéval aux fantaisies obsolètes.

En ce qui concerne La Marquise de Gange, Adélaïde de Brunswick, princesse de Saxe, l'Histoire d'Isabelle de Bavière, reine de France (1813 ${ }^{77}$ ), ou encore le recueil de nouvelles héroïques et tragiques publié sous le titre Les Crimes de l'amour (1799), aucune occurrence n'a été dépistée. Ceci paraît logique, parce que le caractère historique semble déconseiller l'usage de ces antonomases plutôt burlesques. Or, dans le cas des historiettes, contes et fabliaux écrits à la Bastille entre 1785 et 1788, dont certains récits adoptent le style facétieux des nouvelles classiques de Cervantès, l'absence de ces substantifs nous semble, au contraire, surprenante. Pour ce qui est de la correspondance de Sade et de ses proches, elle contient tout autant les noms de Dulcinée et don Quichotte que celui de Sancho Panza, lequel n'apparaît pas dans ses ouvrages romanesques. Quant aux épîtres, même dans les descriptions peu favorables, elles reprennent ces prénoms mais vidés de toute agressivité linguistique, car ceux-ci sont dépourvus de la charge sémantique virulente présente dans les romans. Enfin, bien que le vaste corpus examiné n'apporte qu'un nombre assez réduit d'exemples, tous les échantillons présentés ici permettent d'examiner en détail les quelques échos du Quichotte sous la plume de Sade.

\section{NOTES}

1. Nouvelles de M. de Cervantès, 1707. Nouvelles choisies - $3^{\mathrm{e}}$ tablette, $6^{\mathrm{e}}$ rayon - (Alice M. LABORDE, La bibliothèque du marquis de Sade au château de La Coste, Paris-Genève, Champion-Slatkine, 1991, reg. 350).

2. "Don Quichotte, traduit de l'espagnol par Filleau de St-Martin, 1713-1722. $3^{\mathrm{e}}$ tablette, $8^{\mathrm{e}}$ rayon " (Ibid., reg. 384). Il s'agit de l'Histoire de l'admirable don Quixotte de la Manche (1678) qui fut la première traduction intégrale du roman. Elle fut réimprimée plus de vingt fois entre 1730 et 1781 (Maurice BARDON, El Quijote en Francia en los siglos XVII y XVIII. Traduction de Jaime Lorenzo Miralles, San Vicente del Raspeig, Publicaciones de la Universidad de Alicante, p. 445 et p. 719).

3. Renée-Pélagie Cordier de Launay de Montreuil.

4. Plusieurs ouvrages portant le titre du Nouveau Don Quichotte ayant été publiés à l'époque, il est difficile d'affirmer que celui dont disposait Sade soit l'œuvre de Cervantès. Parmi ces publications, figure en premier lieu Pharsamon ou les Folies romanesques de Marivaux (écrit en 1712 et publié en 1737), qui reçut aussi le nom du Nouveau Don Quichotte, 2 vol., in-12. (Nicolas TOUSSAINT LE MOYNE DESESSARTS, Nouveau dictionnaire bibliographique portatif ou Essai de bibliographie universelle..., Chez N.L.M. Desessarts Imprimeur-Libraire, An VIII-1799, p. 274, [consulté le 15/06/2019], URL : https://book.google.es/books ?id =G1c3YTgTcHQC). Or, si l'on tient compte de la datation de la lettre (1781), il est plus probable que ce soit un ouvrage plus récent, par exemple, Le Nouveau don Quichotte imité de l'allemand de M. Wieland, par Mme d'Ussieux, 
Bouillon, Imp. de la Société Typographique, $1770: 2$ vol. in- $8^{\circ}$ (attribué aussi à Louis Dussieux, son mari), ou, enfin, qu'il s'agisse d'une réimpression de la traduction de Filleau (Histoire de l'admirable Don Quichotte de La Manche, traduite de l'espagnol de Michel de Cervantès, nouvelle édition, revue, corrigée \& augmentée, Rouen, Pierre Machuel ; Lyon, Amable Le Roy, 1781, 6 vol. in-12. Toutefois le problème subsiste parce que le nombre de volumes des différentes options ne coïncide pas avec celui qui est signalé dans la lettre de Renée.

5. Alice M. LABORDE, Correspondances..., op. cit., vol. XVI, Sade au donjon de Vincennes (1781), Genève, Slatkine, 2007, p. 171 et p. 224.

6. Lettre sans date reçue le samedi 22 décembre 1781 (ibid., p. 273).

7. Alice M. LABORDE, Correspondances..., op. cit., vol. XX, Sade à la Bastille (1787-1788), Genève, Slatkine, 2007, p. 151.

8. Nouvelles espagnoles de Michel de Cervantes. Traduction nouvelle, et avec des notes, ornée de figures en taille-douce, 2 vol. , Paris, 1777 (Maurice BARDON, El Quijote en Francia..., op. cit., p. 723).

9. Donatien-Alphonse-François de $\mathrm{SADE}$, Idée sur les romans dans Les crimes de l'amour: nouvelles héroïques et tragiques précédées d'une Idée sur les romans, édition établie et annotée par Éric LE GRANDIC et présentée par Michel Delon, Cadeilhan, Zulma, coll. « Dix-huit », 1995, p. 24-25.

10. En effet, les critiques littéraires ont souligné à maintes reprises le parallélisme entre les techniques et les structures des récits cervantins et sadiens. "Peut-être Justine et Juliette, à la naissance de la culture moderne, sont-elles dans la même position que Don Quichotte entre la Renaissance et le classicisme. [...] Les personnages de Sade lui répondent à l'autre bout de l'âge classique, c'est-à-dire au moment du déclin» (Michel Foucault, Les mots et les choses. Une archéologie des sciences humaines, Paris, Gallimard, 1966, p.222-223). On pourra également consulter Christian HermanN, "L’Espagne du Marquis de Sade ", Mélanges de la Casa de Velázquez, t. 28-2, 1992, p. 71-86 ; Frédéric CONROD, « Histoire de Juliette, ou la nouvelle Don Quichotte : le grand voyage du motif baroque dans le roman moderne », 29 p., Congrès Sade (Fall 2004-Spring 2005), Electronic journal, URL : www.cofc.edu/desade/papers/conrod01.pdf ; Elena DEANDA-CAMACHO, "Quixotic Sade : Echoes of Cervantes in 120 Days of Sodom ", Studies in Eighteenth-Century Culture, vol. 46, 2017, p. 21-33 [10.1353/sec.2017.0004].

11. Des auteurs contemporains se servent aussi de cette figure rhétorique. Maurice BARDON (El Quijote en Francia..., op. cit., p. 889-890) introduit plusieurs cas : Rétif emploie, par exemple, « un don Quichotte» (Tableaux de la bonne compagnie, 1787, t. I, p. 123 et t. II, p. 151), «le don Quichotte " (La dernière aventure d'un homme de quarante-cinq ans, 1909, p. 217) et la locution «à la Sancho Pança » (Les contemporaines, t. XXXIX, nouvelle CCXLVII La belle imprimeuse, p. 215). Mercier parle de "faire le Quichotte" dans sa pièce Jeneval ou le Barnevelt françois (III, 3) et recueille l'expression "donquichotisme » dans sa Néologie, ou Vocabulaire de mots nouveaux, à renouveler, ou pris dans des acceptions nouvelles (t. I, p. 197, an IX [1801], Paris).

12. Au sujet des premières traductions du Quichotte en français, et en particulier de celle réalisée par César Oudin, voir : Marc ZuILI, «Étude introductive » à son édition du Tesoro de las dos lenguas española y francesa/Tresor des deux langues françoise et espagnolle de César Oudin, Paris, Honoré Champion, 2016, p. 101-107.

13. Alexandre CIORANESCU, Le masque et le visage : du baroque espagnol au classicisme français, Genève, Droz, 1983, p. 155-156.

14. «De Dulcinée du Toboso (Dulcinea del Toboso), nom donné par don Quichotte à la dame de ses pensées dans le roman de Cervantes (v. don Quichotte) ». Philibert-Joseph LE Roux, Dictionnaire comique, satyrique, critique, burlesque, libre \& proverbial, Amsterdam, Chez Michel Charles Le Cene, 1718, p. 174.

15. Le Dictionnaire de Trévoux, dans son édition de 1738-1742, contient une seule explication complètement étrangère au domaine littéraire : «Tèrme de Fleuriste. Espéce de tulippe. Elle est d'un blanc de lait, \& couleur de lacque.» (Nancy, Pierre Antoine, 1738-1742, t. II [consulté le 
22/05/2020], URL : http://www.cnrtl.fr/dictionnaires/anciens/trevoux/menu1.php), tandis que, dans son édition de 1771, il conserve cette acception-là et en ajoute une seconde : "C'est le nom qu'on donne dans le style familier \& badin à la maîtresse d'un homme. »

16. "Caractère de Dom Quichote, Héros romanesque et burlesque " (Jean-François FÉRAUD, Dictionnaire critique de la langue française, Marseille, Mossy, 1787-1788, vol.3, [consulté le 06/06/2019], URL : https://www.cnrtl.fr/dictionnaires/anciens/feraud/menu.php)

17. «sub. fém. ou masc. Jument maigre et efflanquée, que l'on donne pour monture à DonQuichotte. On donne aussi ce nom en plaisantant à un cheval ruiné et de mauvaise mine ». Paris, Chez J. J. Smits et Cie., Imp.-Lib.

18. « subst. fém. Terme familier, pour signifier une femme mal bâtie et maussade » (Dictionnaire de l'Académie, ibid.)

19. Grâce aux articles du chevalier de Jaucourt ( Séville ", "La Manche ", « Engagement », « Ridicule (le) », « Roman de chevalerie » et « Worcestershire »), de Diderot (« Arme. Armure »), de D’Alembert ( Continuateurs»), de Lefevbre ( Folie») et d'un collaborateur anonyme ("Chevalier errant»). Cf. Alejandro RAmírez, "Cervantes dans l'Encylopédie», Romance Notes, vol. 12, no 2 (Spring, 1971), p. 407-412.

20. Alice M. LABORDE, Correspondances du Marquis de Sade et de ses proches enrichies de documents, notes et commentaires, vol. XXI, De la Bastille à Charenton (1789). La Libération de Sade (1790), Genève, Slatkine, 1996, note p. 276.

21. SADE, CEuvres, édition établie par Michel Delon, Paris, Gallimard, coll. «Bibliothèque de la Pléiade ", 1990, t. I, p. 734.

22. Notons au passage que Léonore et Sainville sont une pure invention de Filleau de SaintMartin qui, dans sa traduction du Quichotte (celle dont Sade dispose à La Coste), insère deux nouvelles: l'une fait de Léonore la femme du jaloux Osorio; l'autre met en scène les amours malheureuses de Sainville et Sylvie. L'influence de ces personnages dans les histoires que Léonore et Sainville racontent dans Aline et Valcour est considérable.

23. SADE, CEuvres, op. cit., 1990, p. 736-737.

24. Ibid., p. 980.

25. Ibid., p. 1009 note*.

26. Marc-Antoine LAUGIER, Histoire de la République de Venise depuis sa formation jusqu'à présent, Paris, Veuve Duchesne, t. XII, 1768, p. 205-206.

27. SADE, CEuvres, op. cit., 1990, p. 105.

28. Ibid., p. 138.

29. Ibid., p. 105-106.

30. Ibid., p. 138.

31. Ibid., p. 153.

32. Ovide remarquait que les femmes âgées étaient plus savantes que les jeunes filles dans les plaisirs car elles avaient l'expérience qui seule perfectionne tous les talents (cf. L'Art d'aimer, liv. II, v. 663-702).

33. Le Dictionnaire de l'Académie française, dans ses $4^{\mathrm{e}}(1762)$ et $5^{\mathrm{e}}(1798)$ éditions, contient cette acception pour le terme 'emplâtre' : «Il se dit aussi d'une personne qui n'a pas de vigueur d'esprit, \& qui n'est pas capable d'agir », op. cit.

34. SADE, Euvres, op. cit., 1990, p. 153-154.

35. Ibid., p. 153.

36. Ibid., p. 206.

37. Nom que l'on prend en entrant au couvent.

38. Justine avait adopté ce nom pour cacher son identité.

39. Ce mot disparaît dans d'autres versions des Infortunes: "Voilà celle que la société destine à remplacer la dernière partie, mesdemoiselles [...]. Sophie, me dit alors le supérieur, vous êtes la 
plus âgée de la classe, et je vous élève au poste de doyenne » (SADE, Les Infortunes de la vertu. Texte établi sur le manuscrit présenté et annoté par Béatrice Didier, Paris, Gallimard, 1970, p. 191); " [...] cette fille occupe dans une autre tour le même poste que vous occupez dans la vôtre, elle est doyenne » (SADE, Euvres, édition établie par Michel Delon, Paris, Gallimard, coll. « Bibliothèque de la Pléiade ", t. II, 1995, p. 82). Ce problème surgit des différentes corrections faites par Sade et donc de la pluralité des versions d'un même texte. "L'éditeur de manuscrits se trouve devant le même dilemme qu'un architecte chargé de restaurer un bâtiment, plusieurs fois construit et reconstruit. Quelle époque faut-il privilégier?» (SADE, Contes étranges, texte établi, présenté et annoté par Michel Delon, Paris, Gallimard, 2014, p. 319).

40. $\mathrm{SADE}$, Les infortunes de la vertu, présentation par Jean Marie Goulemot, Paris, GarnierFlammarion, 2014 (révision de l'édition de 1969) [consulté le 06/06/2019], URL: https:// books.google.es/ books ?id =TiepCwAAQBAJ.

41. SADE, La Philosophie dans le boudoir, édition présentée, établie et annotée par Yvon Belaval, Paris, Gallimard, 1976. p. 261.

42. Avocat de la ville d'Apt, notaire et procureur qui resta à la tête des affaires de Sade pendant vingt-six ans.

43. Laborde indique dans une note «Dulcinée, sa fille Françoise » (ibid., p. 105) ; Françoise-Pélagie était l'avant-dernière des cinq enfants des Montreuil et, donc, la sœur de Renée. Elle naquit le 12 octobre 1760 et mourut le 9 février 1837. Fin janvier 1783, elle avait épousé le marquis de Wavrin ou Vavrin ( $c f$. Alice M. LABORDE, Correspondances..., vol. xx, op. cit., p. 51-52). Pourtant, dans une autre note de la même auteure, nous trouvons des erreurs lorsqu'elle signale « Il s'agit de Françoise-Pélagie de Sade, sœur cadette de Renée [...]. Elle meurt en 1836 » (Alice M. LABORDE, Correspondances..., op. cit., vol. XVIII, Sade au donjon de Vincennes (1783-1784), Genève, Slatkine, 2007, p. 37). Laborde se trompe car le nom de cette femme, belle-sœur du divin marquis, ne peut pas être 'de Sade' mais, soit 'de Montreuil' - son nom de naissance -, soit 'de Wavrin' - son nom de femme mariée -. C'est Renée qui se maria à Donatien et, donc, la seule sœur qui pût recevoir le nom 'de Sade'.

44. Selon Paul Bourdin, cette «dulcinée » était «[s]ans doute une des sœurs de la marquise et, vraisemblablement, mademoiselle de Launay » (Correspondance inédite..., op. cit, p. 85 n. 1), c'est-àdire Anne-Prospère. « Anne-Prospère de Launay de Montreuil, la sœur cadette de Renée, naquit le 27 décembre 1751 [...]. Elle mourut chez ses parents, à Paris, le 13 juin 1779 » (Alice M. LABORDE, Correspondances..., vol. XVIII, op. cit., note p. 37). Quant à Chantal THOMAs, elle indique que la mort d'Anne-Prospère était survenue le 13 mai 1781 (Sade, Paris, Seuil, 1994, p. 233). Sa fuite en Italie avec son beau-frère s'était produite durant trois mois en 1772, quand elle avait 21 ans. Son père s'appelait Claude-René Cordier de Launay, de Montreuil (Alice M. LABORDE, Correspondances..., vol. xx, op. cit., note p. 78), d'où le premier de ses patronymes.

45. Paul BouRdin transcrit le nom en minuscule (Correspondance inédite du marquis de Sade, de ses proches et de ses familiers, publiée avec une introduction, des annales et des notes, Genève, Slatkine Reprints, 1971 ; réimpression de l'édition de Paris, 1929, p. 85 n. 1).

46. Alice M. LABORDE, Correspondances..., op. cit., vol. VIII, Incarcération de Sade au donjon de Vincennes (1777) [13 février 1777-14 juin 1778], Genève, Slatkine, 1998, p. 104-105.

47. L'avocat du marquis à Aix.

48. Maurice LEVER, Donatien Alphonse François, marquis de Sade. Biographie, Barcelone, Seix-Barral, 1994, p. 271 et p. 277.

49. $\mathrm{M}^{\text {me }}$ Doyen de Baudoin lui écrivit d'autres missives datées du 13 août 1781 et du 12 février 1782.

50. Alice M. LABORDE, Correspondances..., op. cit., vol. XIII, Nouvelle incarcération de Sade au donjon de Vincennes (1778). Genève, Slatkine, 1998, p. 157. 
51. Alice M. LABORDE, Correspondances..., vol. XVIII, op. cit., p. 178.

52. SADE, Cuvres, op. cit., 1990, p. 894.

53. Ibid., p. 830.

54. Aldonse est un vieux prénom provençal convenant aussi bien aux garçons qu'aux filles. Pour restituer la diction provençale, il faut l'orthographier "Aldonze». Voir: https:// gw.geneanet.org/fraternelle?

lang $=$ en\& $=$ de + barrigue + de + montvalon\&oc $=0 \& p=$ aldonse

55. L'abbé Jacques-François-Paul-Aldonce, son oncle paternel (Alice M. LABORDE, Correspondances..., op.cit., vol. XX, note p.90), ainsi qu'une connaissance de la famille que le comte de Sade protégeait (à laquelle il donna une pension et proposa son aide pour lui trouver un travail), portaient ce prénom (Alice M. LABORDE, Correspondances..., op.cit., vol.XIX, Sade à la Bastille [29-02-1784/31-12-1786], Genève, Slatkine, 2007, p. 72).

56. SADE, Les Infortunes de la vertu, op. cit., 1970, p. 259.

57. Paul Bourdin, Correspondance inédite..., op. cit., p. 9.

58. «Ester à droit, comparaître, se présenter devant le juge sur l'assignation qu'on a reçue ; expression de l'ancienne jurisprudence qui ne s'appliquait qu'au droit criminel. » Le Littré (1880). Voir : http://dictionnaire.sensagent.leparisien.fr/ester/fr-fr/

59. Alice M. LABORDE, Correspondances..., vol. VIII, op. cit., p. 256.

60. Il le rédige dans une lettre autographe sur un papier vergé azuré avec filigrane écu ovale, couronné et lauré où deux fleurs de lys apparaissent en chef ( $c f$. Alice M. LABORDE, Correspondances..., vol. XXI, op. cit., note p. 95).

61. Pierre Chenon père occupait le poste de commissaire au Châtelet et était chargé depuis 1774 de la forteresse de la Bastille (Alice M. LABORDE, ibid, note p. 97).

62. Il est vrai que cet engouement disparaîtra après la révolution. Un certificat de résidence du 7 mars 1793 où figurent sa description physique et ses coordonnées nous le prouve car il y est nommé « Louis Sade, homme de lettres » (ibid., note p. 258).

63. Ibid., p. 95.

64. SADE, Euvres, op. cit., 1990, p. 894.

65. SADE, La Philosophie..., op. cit., p. 7.

66. Ibid.

67. Paul BouRdin, Correspondance inédite..., op. cit., p. 64.

68. Les ressemblances entre Sade et don Quichotte pourraient être étendues à certains épisodes de la vie du marquis et de celle de Cervantès. D'après Camilo J. Cela, Cervantès fut « el hombre a quien zurró el destino y derrotó la envidia » [l'homme que le destin avait rossé et qui fut vaincu par la jalousie] (Camilo José CELA, «Amo la palabra », Discurso de 23-04-1995, Entrega Premio Cervantes, Centro Virtual Cervantes, URL :

https://cvc.cervantes.es/literatura/escritores/cela/discursos/discurso_01.htm [consulté le 26/05/2019]). L'affirmation de Cela, dans le discours qu'il prononça lorsque lui fut remis le prix Cervantès, nous fait penser à Sade qui, devenu bouc émissaire à cause de l'audace de son écriture, passa une grande partie de sa vie en prison.

69. Forme provençale de «mademoiselle » et manière affective de nommer Marie-Dorothée de Rousset.

70. Alice M. LABORDE, Correspondances..., op. cit, vol. XIV, Sade au donjon de Vincennes (1779), Genève, Slatkine, 2007, p. 168.

71. Aussi nommé Martin Quiros, Dom Quiros ou La Jeunesse, au service du marquis.

72. En réalité, Filleau, Histoire de l'admirable..., op. cit., parle d'un bassin de barbier (cf. t. I, chap. XX et chap. XXIV, [consulté le 06-06-2019], URL: https://books.google.es/books? id =TKhQzxa0_HcC). 
73. Carteron se trompe car le Chevalier de la Triste-figure n'est autre que Don Quichotte luimême. Il est évident que le valet confond le personnage du roman de Cervantès avec le "Chevalier de la Montagne», autrement appelé le "Chevalier du bois» ou le "Chevalier déchiré » (« el Roto de la Mala Figura » dans le roman espagnol), surnoms du fou Cardenio (t. I, chap. XXII «De ce qui arriva au fameux Don Quichotte dans la Montagne noire » et chap. XXIII : "Où se continue l'aventure de la Montagne noire». La Montagne noire est la Sierra Morena). Voir : https://books.google.es/books ?id =TKhQzxa0_HcC [consulté le 19-06-2019].

74. Lettre du 23 septembre 1779 reçue le 30 (Alice M. LABORDE, Correspondances..., vol. XIV, op. cit., p. 248).

75. Ibid., p. 253-254.

76. Ibid., p. 87.

77. Tous achevés en 1813, même si seul le premier fut publié à cette date-là. Les deux autres ont donné lieu à des éditions posthumes.

\section{RÉSUMÉS}

Cette étude repose sur l'analyse d'un corpus réunissant l'ensemble des romans de Sade ainsi que sa correspondance, d'après les recueils de P. Bourdin (1929) et A. M. Laborde (1991-2007). L'auteur des Lumières connaissait plusieurs ouvrages de Cervantès, écrivain du Siècle d'Or qu'il admirait. Or ce ne fut pas seulement à travers ces lectures que Sade rencontra les personnages créés par le génial écrivain espagnol car, $a u X{ }^{\mathrm{e}}{ }^{\mathrm{e}}$ siècle, des dictionnaires avaient commencé à colliger leurs prénoms devenus noms communs. Puis, au XVIII ${ }^{\mathrm{e}}$ siècle, le Dictionnaire comique de Le Roux, le Dictionnaire critique de la langue française de Féraud et le Dictionnaire de l'Académie française ont conservé ces mêmes substantifs et en ont ajouté quelques autres toujours tirés du Don Quichotte de la Manche. Il est donc possible de trouver des références à Dulcinée et à don Quichotte dans Les 120 Journées de Sodome, Les Infortunes de la vertu, Aline et Valcour et La Philosophie dans le boudoir. Aussi la correspondance de Sade et de ses proches contient-elle les noms de Dulcinée, don Quichotte ou Sancho Panza, soit pour désigner le divin marquis lui-même, soit pour faire allusion à des personnes de son entourage.

In this paper we analyse a corpus of all of Sade's novels as well as his correspondence on the basis of collections by P. Bourdin (1929) and A. M. Laborde (1991-2007). The Age of Enlightenment author was acquainted with several works by Cervantes, a writer of the Spanish Golden Age who he admired. Yet, it was not only through these readings that Sade discovered the characters created by the Spanish author, since in the seventeenth century some dictionaries had started to include those characters' proper names used as common nouns. Later, in the eighteenth century, Le Roux's Dictionnaire comique, Féraud's Dictionnaire critique de la langue française, and the Dictionnaire de l'Académie française kept those same nouns and added some others also drawn from Don Quixote. It is therefore possible to find references to Dulcinea and to Don Quixote in Sade's Les 120 Journées de Sodome, Les Infortunes de la vertu, Aline et Valcour and La Philosophie dans le boudoir. By the same token, some letters written both by Sade and by his friends and family also contain the names of Dulcinea, Don Quixote or Sancho Panza in order to refer to either the Divine Marquis himself or to allude to people in his intimate circle. 
INDEX

Mots-clés : Siècle d'or, Cervantès, Dulcinée, siècle des Lumières, antonomase

Keywords : Spanish Golden Age, Cervantes, Dulcinea, Age of Enlightenment, antonomasia

\section{AUTEUR}

\section{IRENE AGUILÁ-SOLANA}

(Université de Zaragoza - Espagne) 